\title{
An implementation of the Local Ensemble Kalman Filter in a quasi geostrophic model and comparison with 3D-Var
}

\author{
M. Corazza ${ }^{1,2}$, E. Kalnay ${ }^{1}$, and S. C. Yang ${ }^{1}$ \\ ${ }^{1}$ University of Maryland, Department of Meteorology, College Park, MD, USA \\ ${ }^{2}$ ARPAL - CFMI-PC, V.le Brigate Partigiane 2, 16129, Genova, Italy
}

Received: 21 August 2006 - Revised: 16 February 2007 - Accepted: 16 February 2007 - Published: 22 February 2007

\begin{abstract}
We perform data assimilation experiments with a widely used quasi-geostrophic channel model and compare the Local Ensemble Kalman Filter (LEKF) with a 3D-Var developed for this model. The LEKF shows a large improvement, especially in correcting the fast growing modes of the analysis errors, with a mean square error equal to about half that of the 3D-Var. The improvement obtained in the analysis is maintained in the forecasts, implying that the system is capable of correcting the initial errors responsible for later forecast error growth.

Different configurations of the LEKF are tested and compared. We find that for this system, adding random perturbations after every analysis step is more effective than the standard variance inflation in order to avoid underestimating the background error covariance and the consequent filter divergence.

Experiments indicate that optimal results are obtained with a relatively small number of vectors $(\sim 30)$ in the ensemble. The LEKF is characterized by the "localization" of the analysis process over local domains surrounding each gridpoint of the model grid. We find that, when using a fixed number of ensemble vectors, there is an optimal size of the local horizontal domain beyond which the results do not change further.
\end{abstract}

\section{Introduction}

Numerical weather prediction has substantially improved in recent years, due to both an enhancement in the dynamics and physical parameterizations in models, and to new methods to generate initial conditions in the data assimilation processes (e.g. Kalnay, 2003). It is well known that the atmospheric flow is a chaotic system and that numerical forecasts

Correspondence to: M. Corazza

(matteo.corazza@arpal.org) are sensitive to small changes in the initial conditions, with a rapid growth of the initial errors that leads, in a relatively short time, to deviations responsible for limiting the predictability of the flow to a week or so. For this reason, during the last decades a great effort has been devoted to study new methods aimed at improving the description of the predictability of the flow and, as a consequence, of the initial conditions.

Presently, several operational centers use 3-Dimensional Variational data assimilation systems (3D-Var, e.g. Parrish and Derber, 1992; Lorenc, 1986) to generate the analyses required to provide initial conditions to the model. This method is a statistical interpolation between the new observations and a short range forecast (typically 3 to $12 \mathrm{~h}$ ), which is used as first guess or background. In this process, the corrections of the background toward the observations, i.e. the analysis increments, are strongly dependent on the inverse of their error covariances, and only take place within the subspace spanned by the background error covariance. Therefore a good representation of the observation and background error covariances is one of the most important aspects in the framework of the present efforts in developing data assimilation systems. In 3D-Var methods the background error covariance $\mathbf{B}$ is given by a statistical average of the error structures, and is maintained constant in time. That is, there is no accounting for the variations of the atmospheric state and consequent day-to-day variability of the background errors due to the actual state of the flow. These flow-dependent errors are hereafter referred to as the "errors of the day", the importance of which has been described in several recent papers and books (e.g. Courtier et al., 1994; Kalnay, 2003; Corazza et al., 2003).

Kalman filtering (see Daley, 1993), in which $\mathbf{B}$ is predicted $\left(\mathbf{B}_{\mathbf{t}}=\mathbf{L} \mathbf{B}_{\mathbf{t}-\mathbf{1}} \mathbf{L}^{\mathbf{T}}\right.$, where $\mathbf{L}$ is the tangent linear model, $\mathbf{L}^{\mathbf{T}}$ its transpose and $t$ is time) can be considered the natural and complete approach to the problem of representing the variability of $\mathbf{B}$, but the computational cost related to

Published by Copernicus GmbH on behalf of the European Geosciences Union and the American Geophysical Union. 
this formulation makes its operational application impossible without major simplifications, even considering the potential development of the computational facilities during the next decade. There are other methods that try to account for the "errors of the day" in the forecast error covariance, such as the modification of B in 3D-Var (Purser, 2005; De Pondeca et al., 2006; Corazza et al., 2002), 4-dimensional variational data assimilation (4D-Var, e.g. Courtier et al., 1994; Rabier et al., 2000; Klinker et al., 2000), ensemble Kalman Filtering (EnKF, e.g. Houtekamer and Mitchell, 1998; Hamill and Snyder, 2000), and the method of representers (Bennett et al., 1996).

Recently, several studies have been carried out in order to substitute the standard $\mathbf{B}$ in 3D-Var with a more complex covariance matrix, no longer constant, but capable to take into account time dependent flow. In particular, a general method to implement any arbitrary anisotropy, and therefore also flux-dependent anisotropies, has been implemented at NOAA/NCEP, as described by Purser (2005), regarding the theoretical geometrical implementation, and by De Pondeca et al. (2006), concerning different implementations of the filter. All the implementations produced an improvement respect to the standard 3D-Var, further confirming the importance of the inclusion of the errors of the day in the data assimilation process. Corazza et al. (2002) experimented with a simpler method, based on the use of the information provided by an ensemble of bred vectors in $\mathbf{B}$, using the same system as in this work, and also showed significant improvements with respect to $3 \mathrm{D}$-Var.

4D-Var is a powerful approach that reduces the computational cost of Kalman Filtering by estimating the initial conditions such that the model forecast best fits the observations within a data assimilation window. 4D-Var produces the same results as Kalman Filtering under the assumptions of perfect linear model framework, of Gaussian errors, and of a Kalman Filter representation of the background error covariance matrix at the beginning of the computation. Although 4D-Var is computationally affordable with the most powerful available computers, its cost is still high compared to 3D-Var. In addition, it requires the development and maintenance of the adjoint of the model.

Another approximation to Kalman Filtering is Ensemble Kalman Filtering (EnKF), where the background error covariance is estimated by the sample covariance from the background ensemble vectors. As a result it has the advantage of being model independent, i.e. no tangent linear and adjoint model are needed, since EnKF only requires as input an ensemble of forecasts and their corresponding predictions of the observations, and from that it produces a new ensemble of analyses. In recent implementations that allow for the use of a relatively small number of ensemble members, EnKF has been shown to be less computationally expensive than 4D-Var (Evensen, 1994). A number of studies have been carried out with promising results (Houtekamer and Mitchell, 1998; Hamill and Snyder, 2000; Tippett et al., 2002; Ander- son, 2001; Etherton and Bishop, 2004), and the system developed by Houtekamer et al. (2005) has been implemented in Canada to provide initial ensemble perturbations.

Kalnay and Toth (1994) have pointed out that the breeding technique used for ensemble forecasting describes the local pattern of the "errors of the day" and proposed a method to use the information made available by an ensemble of bred vectors to in effect approximate the background covariance matrix. In their work they argued that the similarity between breeding (Toth and Kalnay, 1993, 1997) and data assimilation suggests that the background errors should have local structures similar to those of bred vectors, and this conjecture has been confirmed by Corazza et al. (2003) with the same Quasi-Geostrophic model used in this work.

More recently, based on the results of Patil et al. (2001), Ott et al. (2002, 2004) developed a new system, called Local Ensemble Kalman Filter (LEKF), in which the Kalman Filter equations are solved within the space of the ensemble forecasts, but locally in space. This system has been tested on a simple 40 variables Lorenz model (Ott et al., 2004), and on the NCEP Global Forecasting System (Szunyogh et al., 2004, 2005). It is one of the possible implementations of the square-root ensemble Kalman filters, where the analysis increments and errors are computed using a background error covariance derived from the ensemble of vectors, so that the increments lay in the subspace spanned by these vectors. This gives the major advantage of capturing the dominant error growing directions, thus providing a large improvement to the informations available from the observational errors. Other square-root filters derive their computational efficiency from the assimilation of one observation at a time (Tippett et al., 2002), whereas the LEKF computes the analysis independently at each grid point, using all the observations available within a local volume surrounding it. This is advantageous in the presence of a large number of observations, such as satellite data, and it allows for a very efficient parallel implementation.

In this work we test different configurations of the LEKF by implementing it on a quasi-geostrophic channel model described in Rotunno and Bao (1996). The results are compared with those obtained with an optimized 3D-Var system developed by Morss (1998). A perfect model framework is assumed, so that the conclusions of this work are not necessarily valid for a more complex model with errors. However this assumption allows us to explicitly define the "true state of the atmosphere" (by integrating the model from a given initial state) and therefore perform a direct comparison in which the analysis and forecast errors are explicitly represented.

The outline of the work is as follows. In Sect. 2 the implementation of the quasi-geostrophic model and of the 3DVar data assimilation system are briefly reviewed. In Sect. 3, a description of the implementation of the Local Ensemble Kalman Filter is presented. Results are discussed in Sect. 4, and a summary and conclusions close the work. 


\section{Model and 3D-Var data assimilation system}

This study uses the model developed by Rotunno and Bao (1996) and used by Morss (1998) and Morss et al. (2001) to develop a 3D-Var scheme. It is a quasi-geostrophic midlatitude, $\beta$-plane, finite-difference channel model, periodic in longitude, and with impermeable walls on the north and south boundaries. The bottom and the top boundaries are treated as rigid lids. It has no orography, land-sea contrast or seasonal cycle. Quasi-geostrophic potential vorticity is conserved except for Ekman pumping at the surface, $\nabla^{4}$ horizontal diffusion and forcing by relaxation to a baroclinically unstable zonal mean state. All the experiments described in this work have been performed over a domain corresponding to an area of roughly $16000 \times 8000 \times 9 \mathrm{~km}$ with a grid composed by 64 points east-west, 32 south-north and 5 vertical interior layers, plus the bottom and top of the domain, where potential temperature is forecasted to provide vertical boundary conditions for the streamfunction.

As indicated in the introduction, the "true" state of the atmosphere is created by a long integration of the model. The 3D-Var data assimilation system used in this work as the reference data assimilation scheme is based on the code of the scheme originally described in Morss (1998). "Rawinsonde" observations of $u, v$ and $T$ are obtained from the true state of the atmosphere at a fixed number of grid-points in order to avoid interpolation errors. Random Gaussian noise is generated and added to the observations to simulate observational errors compatible with those of the real radiosonde stations of the global operational network.

Analyses are performed every $12 \mathrm{~h}$ and the $12 \mathrm{~h}$ forecast is used as background for the 3D-Var. The system has been optimized for the number of stations used in this work by tuning the amplitude of the background covariance matrix $\mathbf{B}$ originally developed by Morss (1998) so as to minimize the analysis and forecast errors of the 3D-Var. The optimization has been limited to a careful tuning of the amplitude of $\mathbf{B}$, because Morss (1998) showed that the spectral coefficients of $\mathbf{B}$ obtained for the original system are not sensitively dependent of the density of the observational network.

In the original formulation Morss (1998) defines the observation operator $\mathbf{H}$ as the operator that transforms the model variables (grid point components of the potential vorticity and potential temperature) into "rawinsonde observations". The global spectral transforms for the Poisson equation solver and its inverse (required to transform model potential vorticity into observed winds and temperatures and vice versa) are conveniently computed using a global spectral approach within the 3D-Var. As we will see in the next section, this method cannot be directly applied in the framework of the LEKF formulation, since the analysis is computed on local domains, and a different approach has been developed.
3 The implementation of the Local Ensemble Kalman Filter data assimilation system

\subsection{The localization}

The implementation of the Local Ensemble Kalman Filter has been performed following Ott et al. (2002). In the LEKF the representation of the state of the flow is decomposed over local domains around each grid point. A detailed description of the LEKF can be found in Ott et al. (2004) so here we only provide an overview of the approach, following the notation adopted in their paper.

In the 2-dimensional horizontal grid of the model, the state of the flow may be represented by means of a vector field $\boldsymbol{x}(\boldsymbol{r} ; t)$ where $\boldsymbol{r}$ runs over the grid points $\boldsymbol{r}_{m n}$. At each grid point $\boldsymbol{x}$ is a vector of the state variables of the model (in our case potential vorticity and temperature) at all vertical levels. Let $U$ denote the dimension of $\boldsymbol{x}(\boldsymbol{r} ; t)$ at a fixed $\boldsymbol{r}$. In the framework of the QG-model used in this work, where the forecast variables are potential vorticity in the five internal layers and potential temperature at the top and the bottom levels, $U=7$. However, as discussed in Sect. 3.5, the method adopted to locally handle the observation operator requires instead the alternate use of $u, v, T$ at all levels, so that in the implementation used in this work $U$ (in observation space) is equal to 21 . We could have also localized in the vertical, as done in Szunyogh et al. (2005), but because of the low vertical resolution we have included a full column in each local volume.

Following the work of Patil et al. (2001) and Ott et al. (2002), we introduce at each grid point a local vector $\boldsymbol{x}_{m n}$ defined as $\boldsymbol{x}\left(\boldsymbol{r}_{m+m^{\prime}, n+n^{\prime}}, t\right)$ for $-l \leq\left(m^{\prime}, n^{\prime}\right) \leq l$. That is, $\boldsymbol{x}_{m n}(t)$ contains the information of the state of the model over the local area within a $(2 l+1)$ by $(2 l+1)$ sub-grid points centered at $\boldsymbol{r}_{m n}$, with a dimension equal to $(2 l+1)^{2} U$. Considering an ensemble of $k$ forecasts started from the previous analysis step, it is possible to construct the local vectors associated with each member of the ensemble (denoted by $\boldsymbol{x}_{m n}^{b}$, where the superscript $b$ stands for "background"). As in Ott et al. (2002), $\boldsymbol{x}_{m n}^{b}$ at time $t$ can be described by a probability distribution function $F_{m n}\left(\boldsymbol{x}_{m n}^{b}, t\right)$, approximated by a Gaussian distribution identified by a local background error covariance matrix $\mathbf{P}_{m n}^{b}$ and the most probable state $\overline{\boldsymbol{x}}_{m n}^{b}$. Assuming that the dimension of the null space of $\mathbf{P}_{m n}^{b}$ is large compared with the $k$ dimensional subspace orthogonal to the null space, and since $\mathbf{P}_{m n}^{b}$ is symmetric, it is possible to compute an orthonormal set of $k$ eigenvectors $\left\{\boldsymbol{u}_{m n}^{(j)}(t)\right\}$ with a corresponding set of $k$ non-negative eigenvalues $\left\{\lambda_{m n}^{(j)}(t)\right\}$ generally distinct and such as $\lambda_{m n}^{(j)}(k)>0$.

In terms of $\boldsymbol{u}_{m n}^{(j)}$ and $\lambda_{m n}^{(j)}$ the covariance matrix is given by

$\mathbf{P}_{m n}^{b}(t)=\sum_{j=1}^{n} \tilde{\boldsymbol{x}}_{m n}^{b(j)}(t)\left(\tilde{\boldsymbol{x}}_{m n}^{b(j)}(t)\right)^{T}$ 
where

$\tilde{\boldsymbol{x}}_{m n}^{b(j)}(t)=\sqrt{\lambda_{m n}^{(j)}(t)} \boldsymbol{u}_{m n}^{(j)}(t)$.

Working in the $k$ dimensional space $\mathbf{S}_{m n}$ spanned by the vectors $\left\{\boldsymbol{u}_{m n}^{(j)}(t)\right\}$ is particularly advantageous since it is possible to represent $\mathbf{P}_{m n}^{b}(t)$ as a diagonal matrix equal to

$\hat{\mathbf{P}}_{m n}^{b}(t)=\operatorname{diag}\left[\lambda_{m n}^{(1)}, \lambda_{m n}^{(2)}, \ldots, \lambda_{m n}^{(k)}\right]$,

so that its inverse is trivial (here and in the following text, the hat represents vectors or matrices in the $\mathbf{S}_{m n}$ space).

Patil et al. (2001), used 30-pairs of ensembles of bred vectors from the NCEP system (Toth and Kalnay, 1993, 1997) and found that forecast errors in the mid-latitude extra-tropics tend to lie in a low dimensional subset of the $(2 l+1)^{2} U$ dimensional local vector space. For the system used in this work, this result was also very apparent in Corazza et al. (2003). Thus, it is possible to make the hypothesis that the dimension of $\mathbf{S}_{m n}$ is rather low, and that the error variance in all other directions is negligibly small compared to the variance

$$
\sum_{j=1}^{k} \lambda_{m n}^{(j)}
$$

in the directions $\boldsymbol{u}_{m n}(j), j=1,2, \ldots, k$.

\subsection{Data assimilation}

Following Ott et al. (2002), let $\boldsymbol{x}_{m n}^{a}$ denote the random variable (analysis) at time $t$ representing the local vector after using the observations. If $\hat{\boldsymbol{y}}_{m n}^{o}(t)$ is the vector of observations within the local region, with errors normally distributed with covariance matrix $\hat{\mathbf{R}}_{\mathbf{m n}}(\mathbf{t})$, the probability distribution of $\boldsymbol{x}_{m n}^{a}$ is also Gaussian, and is identified by the most probable state $\overline{\boldsymbol{x}}_{m n}^{a}$ and the associated covariance matrix $\mathbf{P}_{m n}^{a}(t)$. The data assimilation step determines $\overline{\boldsymbol{x}}_{m n}^{a}$ (the local analysis) and $\mathbf{P}_{m n}^{a}(t)$ (the local analysis covariance matrix). Similarly to a global 3D-Var data assimilation system, the solution to this problem can be written in the ensemble subspace $\mathbf{S}_{m n}$ as follows

$$
\begin{aligned}
\hat{\overline{\boldsymbol{x}}}_{m n}^{a}(t)= & \hat{\boldsymbol{x}}_{m n}^{b}(t)+\left\{\hat{\mathbf{P}}_{m n}^{b^{-1}}(t)+\hat{\mathbf{H}}_{m n}^{T} \hat{\mathbf{R}}_{m n}^{-1} \hat{\mathbf{H}}_{m n}\right\}^{-1} \times \\
& \hat{\mathbf{H}}_{m n}^{T} \hat{\mathbf{R}}_{m n}^{-1}\left(\hat{\boldsymbol{y}}_{m n}^{o}(t)-\hat{H}_{m n} \hat{\boldsymbol{x}}_{m n}^{b}(t)\right)
\end{aligned}
$$

and

$\hat{\mathbf{P}}_{m n}^{a}(t)=\left[\hat{\mathbf{P}}_{m n}^{b^{-1}}(t)+\hat{\mathbf{H}}_{m n}^{T} \hat{\mathbf{R}}_{m n}^{-1} \hat{\mathbf{H}}_{m n}\right]^{-1}$

where $\hat{\mathbf{H}}_{m n}$ (assumed to be linear) is the local observation operator which maps the local vector $\hat{\boldsymbol{x}}_{m n}(t)$ to the local observations $\hat{\boldsymbol{y}}_{m n}^{o}(t)$. Going back to the local space representation, we have

$\overline{\boldsymbol{x}}_{m n}^{a}(t)=\mathbf{Q}_{m n}(t) \hat{\overline{\boldsymbol{x}}}_{m n}^{a}(t)$
$\mathbf{P}_{m n}^{a}(t)=\mathbf{Q}_{m n}(t) \hat{\mathbf{P}}_{m n}^{a}(t) \mathbf{Q}_{m n}^{T}(t)$

where $\mathbf{Q}_{m n}$ is the $(2 l+1)^{2} U$ by $k$ matrix:

$\mathbf{Q}_{m n}(t)=\left\{\boldsymbol{u}_{m n}^{(1)}(t)\left|\boldsymbol{u}_{m n}^{(2)}(t)\right| \ldots \mid \boldsymbol{u}_{m n}^{(k)}(t)\right\}$.

\subsection{Updating the background field}

The analysis information $\mathbf{P}_{m n}^{a}$ and $\overline{\boldsymbol{x}}_{m n}^{a}$ can be used to obtain an ensemble of global analysis fields $\left\{\boldsymbol{x}^{a(i)}(\boldsymbol{r}, t)\right\}$; $i=1,2, \ldots, k$ as described below (see Ott et al., 2002). These fields represent the ensemble of initial conditions for the atmospheric model. By integrating the ensemble of global fields forward in time to the next analysis time $t+\Delta t$, it is possible to obtain the background ensemble $\left\{\boldsymbol{x}^{b(i)}(\boldsymbol{r}, t+\Delta t)\right\}$. This completes the analysis cycle which, if the procedure is stable, can be repeated for as long as desired. Thus, at each analysis step a global initial condition is available to be used to compute forecasts of the desired duration.

The remaining task is to specify the global analysis fields for the members of the ensemble $\left\{\boldsymbol{x}^{a(i)}(\boldsymbol{r}, t)\right\}$, starting from the analysis information, $\mathbf{P}_{m n}^{a}$ and $\overline{\boldsymbol{x}}_{m n}^{a}$. Let

$\boldsymbol{x}_{m n}^{a(i)}(t)=\overline{\boldsymbol{x}}_{m n}^{a}(t)+\delta \boldsymbol{x}_{m n}^{a(i)}(t)$

denote $k$ local analysis vectors, where

$\sum_{i=1}^{k} \delta \boldsymbol{x}_{m n}^{a(i)}(t)=0$

and

$\mathbf{P}_{m n}^{a}=(k-1)^{-1} \sum_{i=1}^{k} \delta \boldsymbol{x}_{m n}^{a(i)}(t)\left[\delta \boldsymbol{x}_{m n}^{a(i)}(t)\right]^{T}$.

By Eq. (11), the local analysis state $\overline{\boldsymbol{x}}_{m n}^{a}(t)$ represents the mean over the local analysis ensemble $\left\{\boldsymbol{x}^{a(i)}(t)\right\}$, and, by Eq. (12) the error covariance matrix is computed from the vectors $\left\{\delta \boldsymbol{x}^{a(i)}(t)\right\}$. The vectors $\delta \boldsymbol{x}_{m n}^{a(i)}(t)$ can be represented in the form

$\delta \boldsymbol{x}_{m n}^{a(i)}(t)=\mathbf{Y}_{m n}(t) \delta \boldsymbol{x}_{m n}^{b(i)}(t)$,

where the matrix $\mathbf{Y}_{m n}(t)$ can be thought of as a generalized "rescaling" of the original background fields and $\delta \boldsymbol{x}_{m n}^{b(i)}(t)$ are defined as

$\delta \boldsymbol{x}_{m n}^{b(i)}(t)=\boldsymbol{x}_{m n}^{b(i)}(t)-\overline{\boldsymbol{x}}_{m n}^{b}(t)$

and are such that

$\mathbf{P}_{m n}^{b}(t)=(k-1)^{-1} \sum_{i=1}^{k} \delta \boldsymbol{x}_{m n}^{b(i)}(t)\left(\delta \boldsymbol{x}_{m n}^{b(i)}(t)\right)^{T}$.

There are many possible choices for $\mathbf{Y}_{m n}(t)$ due to the nonuniqueness of the square root of matrices. Here we follow the choice made by Ott et al. (2004) making the hypothesis that the original background fields are a good representation of a physical state, so as to minimize the difference 


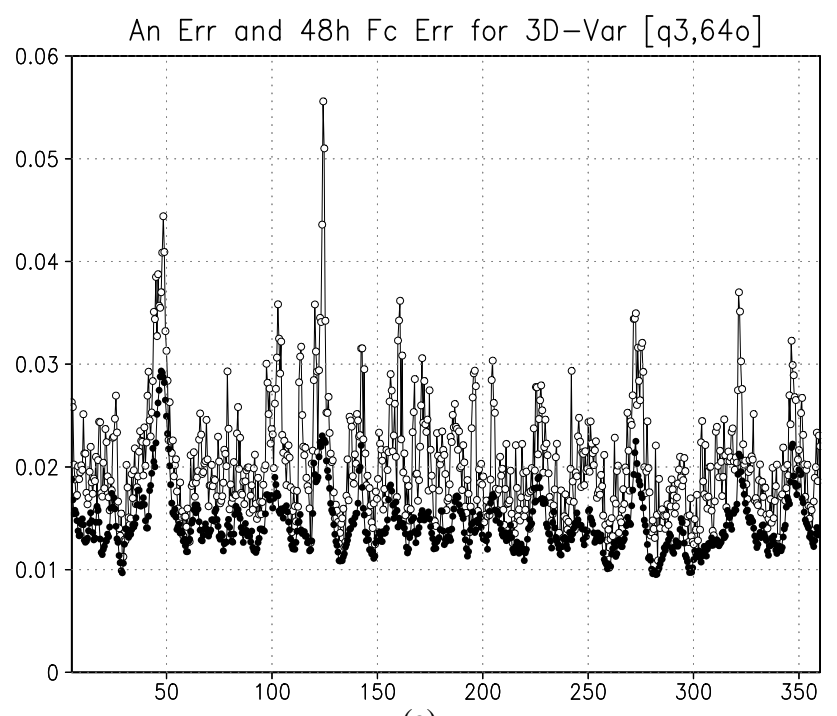

(a)

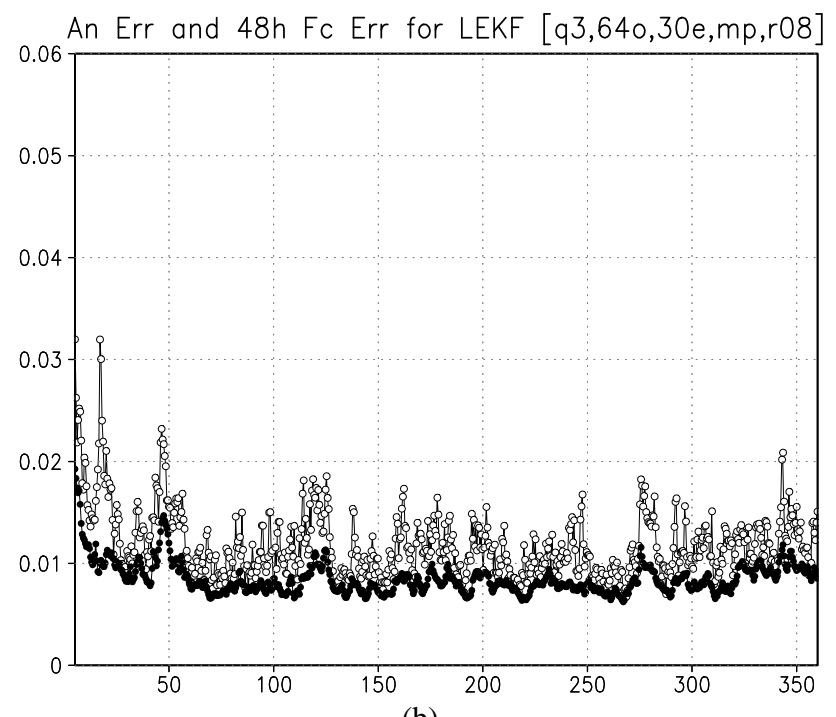

(b)

Fig. 1. (a) Time evolution of Analysis Error (rms) of potential vorticity at mid-level for the regular 3D-Var system (filled dots). The empty dots line represents the $48 \mathrm{~h}$ forecast error. (b) Same as (a) for the reference LEKF system. Here as in the other figures, variables are nondimensionalized following the relations described, for instance, in Rotunno and Bao (1996, page 1058, Sect. 3.c).

between the new analysis and the background ensembles. As a result the solution for $\mathbf{Y}_{m n}(t)$ is:

$$
\begin{aligned}
& \mathbf{Y}_{m n}= \\
& {\left[\mathbf{I}+\mathbf{X}_{m n}^{b^{T}}\left(\mathbf{P}_{m n}^{b}\right)^{-1}\left(\mathbf{P}_{m n}^{a}-\mathbf{P}_{m n}^{b}\right)\left(\mathbf{P}_{m n}^{b}\right)^{-1} \mathbf{X}_{m n}^{b}\right]^{\frac{1}{2}}}
\end{aligned}
$$

where

$\mathbf{X}_{m n}^{b}=(k-1)^{-\frac{1}{2}}\left\{\delta \boldsymbol{x}_{m n}^{b(1)}\left|\delta \boldsymbol{x}_{m n}^{b(2)}\right| \ldots \mid \delta \boldsymbol{x}_{m n}^{b(k)}\right\}$

We can therefore build an ensemble of global fields $\left\{\boldsymbol{x}^{a(i)}(\boldsymbol{r}, t)\right\}$ that can be propagated forward in time to the next analysis time. For this purpose it is possible to set

$\boldsymbol{x}^{a(i)}\left(\boldsymbol{r}_{m n}, t\right)=\mathbf{J} \boldsymbol{x}_{m n}^{a(i)}(t)$,

where $\mathbf{J}$ maps the $(2 l+1)^{2} U$ dimensional local vector to the $U$ dimensional vector updating the vertical profile of the model $(U=7)$ at the grid point $\boldsymbol{r}_{m n}$ at the center of the patch. Equation (18) is applied at every grid point $(m, n)$ of the atmospheric model. Thus Eq. (18) defines an ensemble of $k+1$ global analysis fields $\boldsymbol{x}^{a(i)}(\boldsymbol{r}, t)$ for $i=1,2, \cdots, k$.

\subsection{Handling the stability of the system}

It is known that a frequent problem that can arise from an ensemble of vectors not globally orthogonalized is its tendency to collapse toward a subspace that is too small. As a result, even for a perfect model, the background error covariance tends to be underestimated. These effects will tend to underestimate the forecast error, and therefore to give too little weight to the observations, which can lead to the divergence of the filter (Anderson, 2001). In order to avoid this problem, two different methods aimed at separating and enlarging the ensemble perturbations have been tested and compared in the implementation of the Local Ensemble Kalman Filter.

1. Multiplicative inflation: the amplitude of each perturbation vector is increased by a factor larger than 1 . By applying this method no change in direction is added at the analysis step, but the amplitude of the forecast perturbation vectors is kept sufficiently large (Anderson, 2001).

2. Additive random perturbations: a global $\mathbf{H}^{T}$ operator is applied to a set of $k$ random perturbations vectors in the observational space with an amplitude comparable with the errors of the observations. The resulting fields are added to the global vectors of the ensemble. This allows to "refresh" the ensemble at every analysis step (Annan, 2004), by introducing perturbations in new random directions and therefore increasing the Local Ensemble Dimension (Patil et al., 2001; Oczkowski et al., 2005), which represents the effective number of independent directions present in an ensemble. This method (Corazza et al., 2002) effectively avoids the excessive convergence of bred vectors into a small dimensional space (Wang and Bishop, 2003), and is therefore an alternative solution to the rank-deficiency problem that causes filter divergence. We note that in practice this additive inflation can be implemented without requiring the use of the transpose of the observation operator (e.g., Hunt, 2005). 


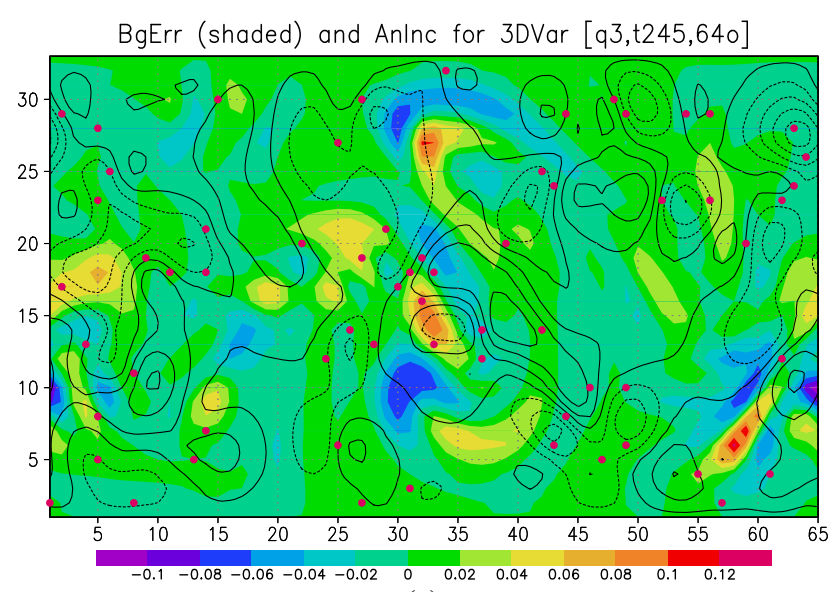

(a)

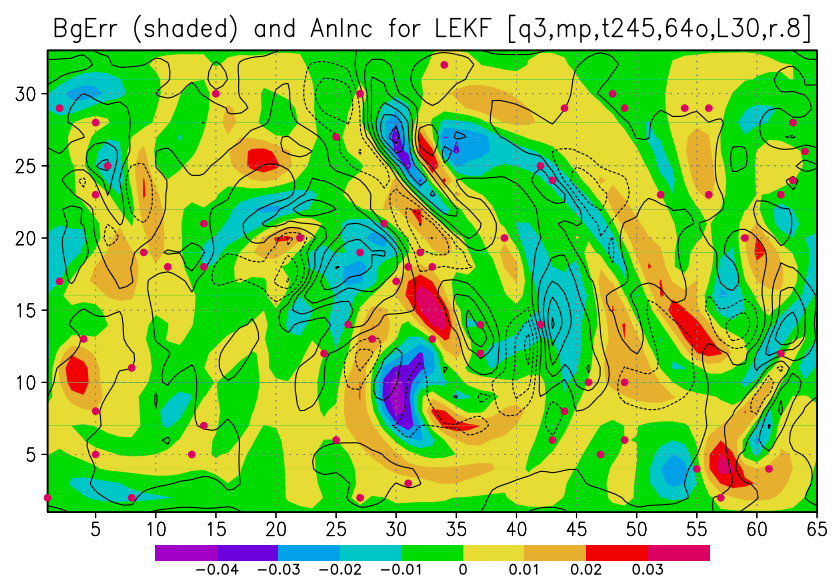

(b)

Fig. 2. (a): Shaded: example of background error for potential vorticity at mid-level at a fixed time $($ day $=123)$ for the regular 3DVar system. The contour plot represents the analysis increments and the red dots indicate the position of the radiosonde stations. (b): same as (a) but for the LEKF. Note different scales in the two figures.

3. Methods 1) and 2) can be used at the same time, in what we call "the combined system".

\subsection{Handling the Poisson Solver in the local domain}

As mentioned in Sect. 2, the observation operator $\mathbf{H}$ performs the transformation from potential vorticity and potential temperature in the model space to horizontal wind velocity and temperature in the observation space. The introduction of $\mathbf{H}$ and $\mathbf{H}^{T}$ requires the solution of a Poisson equation that in the original system used by Morss (1998) is solved by means of the use of global spectral transforms with two boundary conditions: (1) periodic solution in the zonal direction and (2) rigid lateral boundaries at the northern and southern walls. When we work locally, as in the LEKF, this global spectral approach is not feasible. A solution to this problem is found by splitting the observation operator into two parts

$$
\mathbf{H}=\overline{\mathbf{H}} \tilde{\mathbf{H}}
$$

where $\overline{\mathbf{H}}$ is the operator mapping $u, v$ and $T$ defined on the model grid to the observation locations, and $\tilde{\mathbf{H}}$ is the operator transforming potential vorticity and potential temperature into $u, v$ and $T$ over the global model grid.

Localizing $\overline{\mathbf{H}}$ is trivial, since only an interpolation process has to be carried on. In our experiments, this process is even simpler, since the observation locations are on the grid points. Thus it is possible to apply $\tilde{\mathbf{H}}$ once, at the beginning of the data assimilation step, to all the vectors, in order to compute global vectors for $u, v$ and $T$ over the model domain. The computation of the new analysis and perturbations can therefore be carried on considering these as the model variables. At the end of the process the new global vectors in $u, v$, and $T$ are transformed back to the potential vorticity and potential temperature fields needed by the quasi-geostrophic model.

Table 1 summarizes the parameters used for the configuration of the LEKF. The optimal parameters shown in this table are used unless otherwise noted.

\section{Results}

\subsection{Comparison between the 3D-Var and the LEKF}

The time evolution of the analysis root mean square error of the potential vorticity as well as of the $48 \mathrm{~h}$ forecast error for the optimized version of the 3D-Var scheme is presented in Fig. 1a, plotted every $12 \mathrm{~h}$ for about a year (711 values). The system is stable in time, even though the presence of particularly large maxima (e.g. around days 50 and 120) indicate significant variability of the errors in time. This is an example of the importance of the "errors of the day", i.e., the variability of the errors in space and time discussed in the introduction that can be considered an intrinsic property of the stability of the flow. The results shown in Fig. 1a are consistent with those obtained using the original 3D-Var system developed for this quasi-geostrophic model (see Morss, 1998).

The correspondence of large forecast errors with peaks in the analysis errors valid at the same time is a clear indication that these errors are linked to the most unstable modes of the flow. This implies that their correction is important not only to reduce the amplitude of the fluctuations of the analysis errors but also to delay the fast growth of the most unstable (and therefore most important) errors of the flow. The corresponding results for LEKF (Fig. 1b), discussed in the next subsection, clearly indicate that most large "errors of the day" are eliminated.

The Local Ensemble Kalman Filter implementation (Sect. 3) uses the same observational settings as the optimized 3D-Var system. Unless otherwise noted, the number 
Table 1. List of the optimal settings LEKF system used for the simulations presented in this work unless otherwise noted. In particular we have adopted a local domain characterized by an horizontal base of $7 \times 7$ points $(l=3)$, with no vertical localization ( 7 layers). Since the data assimilation variables are $u, v$, and $T, U=21$ (number of variables in a column, Sect. 3.1). Reference simulations have been performed using 30 ensemble members $(k=30)$, and constructing the final global fields as an average of all the analysis fields available from different local domains ("multi column" method, Sect. 4). The average amplitude of the random perturbations added to the ensemble members at the end of the analysis step is $5 \%$ of the average amplitude of the ensemble vectors (Sects. 3.4 and 4). Finally, when multiplicative inflation is used in the combined method, the ensemble vectors are multiplied by a factor equal to 1.02 at the end of the data assimilation step (Sect. 3.4).

$\begin{array}{ll}\text { Horizontal grid } & 64 \times 32 \text { points } \\ \text { Vertical levels } & 5 \text { plus top and bottom } \\ \text { Horizontal dimension of the local domain } & l=3 \\ \text { Number of variables in a column }(u, v, \text { and } T \text { in } 7 \text { layers) } & U=21 \\ \text { Number of members in the ensemble } & k=30 \\ \text { Method to update the global field } & \text { average ("multi column") } \\ \text { Amplitude of the random perturbations } & \sim 5 \% \text { of the average amplitude of the } \\ & \text { ensemble vectors } \\ \text { Inflation factor for the combined system } & 1.02\end{array}$

of ensemble members is equal to 30 . Two methods for updating the new global vectors were tested (see Sect. 3.3). In the first one only the central column of the local domain is updated, so that each point of the global domain is modified only once ("central column method"). In the second method all the points within the domain of each local assimilation are updated, so that the final analysis global vector is represented by an average of the $(2 l+1)^{2}$ values ("multi column method"). Only results for the multicolumn method are presented since they were slightly better than those of the central column method.

Several tests were performed using different values of multiplicative inflation, and we found that this method was initially able to improve significantly the simulation results (during the first 100 analysis steps). However, a slow increase of the error was observed in the second part of the simulation for a wide range of inflation values, suggesting that, at least for this system, inflation alone is not enough to keep the dimension of the space spanned by the ensemble vectors sufficiently large. This result is not necessarily representative of the behavior of a real, more complex, system (e.g. Szunyogh et al., 2005) where multiplicative inflation was satisfactory. In the present quasi-geostrophic system it is possible that the amplitude of the vectors is sufficiently small to keep their behavior close to linear. Under this assumption, the inflation method gives more weight to the observations, but only within the same subspace of the ensemble perturbations, which with time may become too small.

A marked improvement in the stability of the LEKF with time was obtained by the use of additive random perturbations instead of inflation. Unless otherwise noted, all the results shown hereafter are obtained using this method. Tests using the combination of additive perturbations and inflation were slightly better compared to those of the random pertur-

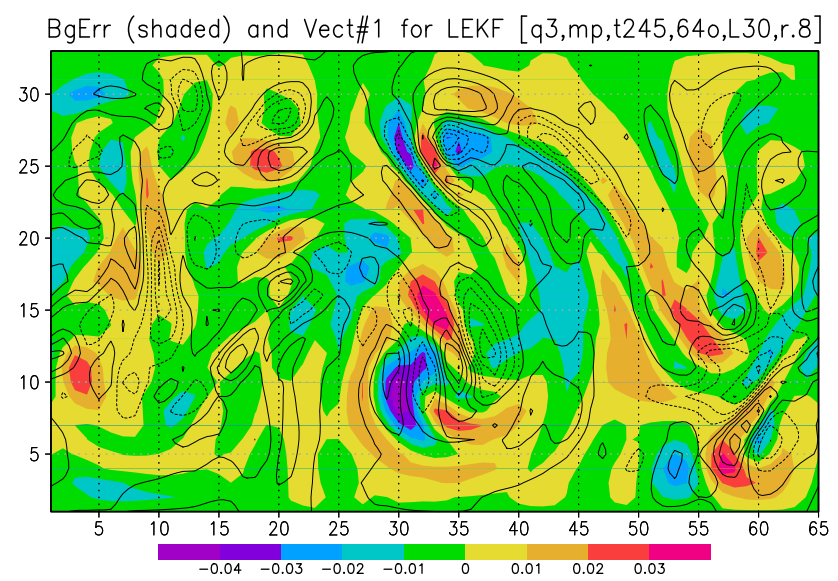

Fig. 3. Shaded: example of background error for potential vorticity at midlevel at the same time of Figs. $2 a$ and $b \quad(d a y=123)$ for the LEKF system. The contour plots represent one vector of the ensemble.

bations alone. A similar strategy was found optimal in Yang et al. $(2007)^{1}$.

Optimal results have been obtained by adding random perturbations to the analyses at the observation points following the procedure described in Sect. 3.4. The standard deviation of the perturbations is equal to about $5 \%$ of the average amplitude of the ensemble vectors. Since the average error of the analysis decreases with the density of the observational network, and the amplitude of the ensemble vector spread de-

\footnotetext{
${ }^{1}$ Yang, S. C., Corazza, M., Carrassi, A., Kalnay, E., and Miyoshi, T.: Comparison of ensemble-based and variational-based data assimilation schemes in a quasi-geostrophic model, in preparation, 2007
} 


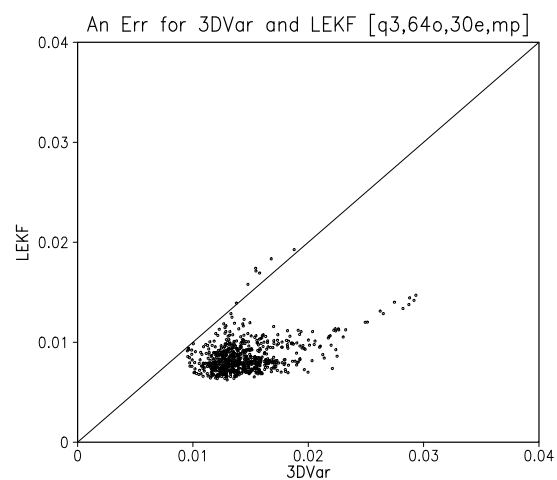

(a)

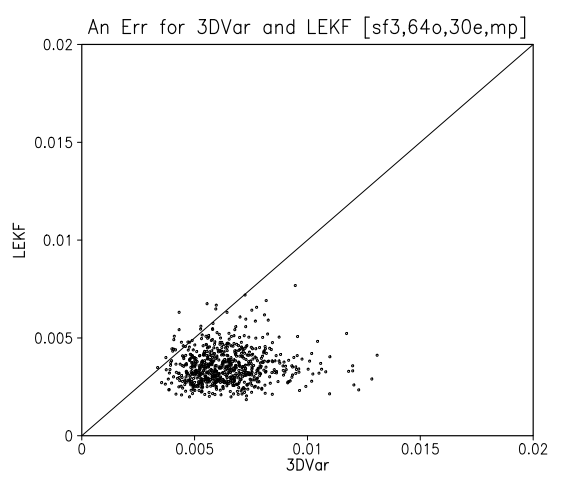

(b)

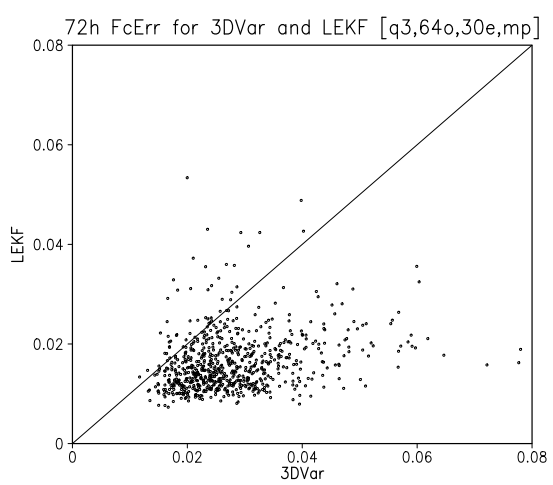

(c)

Fig. 4. (a): Scatterplot of Analysis Error of potential vorticity at mid-level for the regular 3D-Var and for the LEKF. (b): same as (a) for streamfunction. (c): same as (a) for the $72 \mathrm{~h}$ forecast.

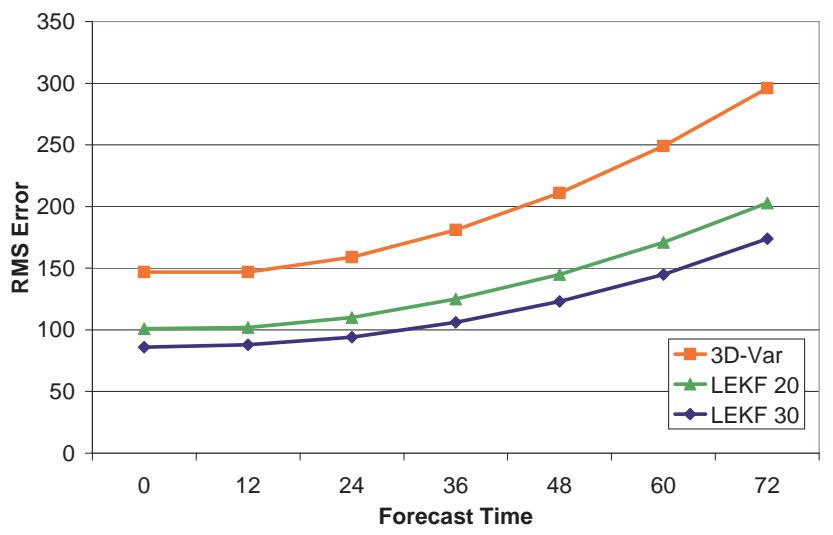

Fig. 5. Forecast evolution of the Average Squared Error for the 3DVar and the LEKF system.

creases as well, it might be thought that in order to keep the system optimal, the amplitude of the random perturbations also needs to change with observational density. However, an important result of this work is that the ratio between the amplitudes of the optimal random perturbations and the ensemble vector spread does not depend on the observational density and is equal to about $5 \%$.

As indicated before, Fig. 1b shows the same analysis and 2-day forecast errors as Fig. 1a but for the LEKF system. Compared to 3D-Var, there is a marked improvement in both the analysis and the forecasts. In particular, the analysis error peaks are reduced, with the consequence that the corresponding forecast errors are much smaller as well. As will be discussed further, this indicates that the LEKF system is capable of correcting the errors lying in the directions of the fast growing modes.

Figure 2a shows an example of background errors (color shaded) for potential vorticity at mid-level obtained by means of the regular 3D-Var scheme. The corresponding analysis increment is shown by contours. The location of the rawinsonde observations is marked by red dots. This time $($ day=123) has been chosen because it is representative of a relatively large error maxima both for the 3DVar and the LEKF systems (see Figs. 1a and b, respectively). As expected, the analysis increments generally correct the errors according to the information provided by the observations, with the amplitude of the increments decreasing with the distance to the observation. However, the 3D-Var corrections do not take into consideration the shape of the background error, since the constant 3D-Var background error covariance matrix is built as the time average of many error estimations.

The performance of the LEKF in computing the analysis increments is shown in Fig. 2b. As anticipated at the beginning of this section, the representation of the background covariance matrix by means of the ensemble of vectors allows the creation of analysis increments with a shape similar to that of the background error. This is in contrast to 3D-Var where the analysis increments are isotropic rather than stretched toward the errors of the day, because the background error covariance matrix has been obtained from a time average of estimated forecast errors (the "NMC method" of Parrish and Derber, 1992). In the LEKF the matrix is locally built from the vectors of the ensemble, each of which contributes to the estimation of the errors of the day. This can be seen in Fig. 3, where one of the vector perturbations after a data assimilation step is plotted on top of the background error. The similarity of the analysis increment shown in Fig. $2 b$ with this vector is apparent.

Figure 4a is a scatterplot of the space averaged analysis errors at midlevel for the LEKF and the 3D-Var respectively. It shows a consistent reduction in the LEKF analysis errors compared to 3D-Var. Most of the large errors from 3D-Var are reduced or completely removed. The only exception in this figure, where errors from the two schemes have similar 

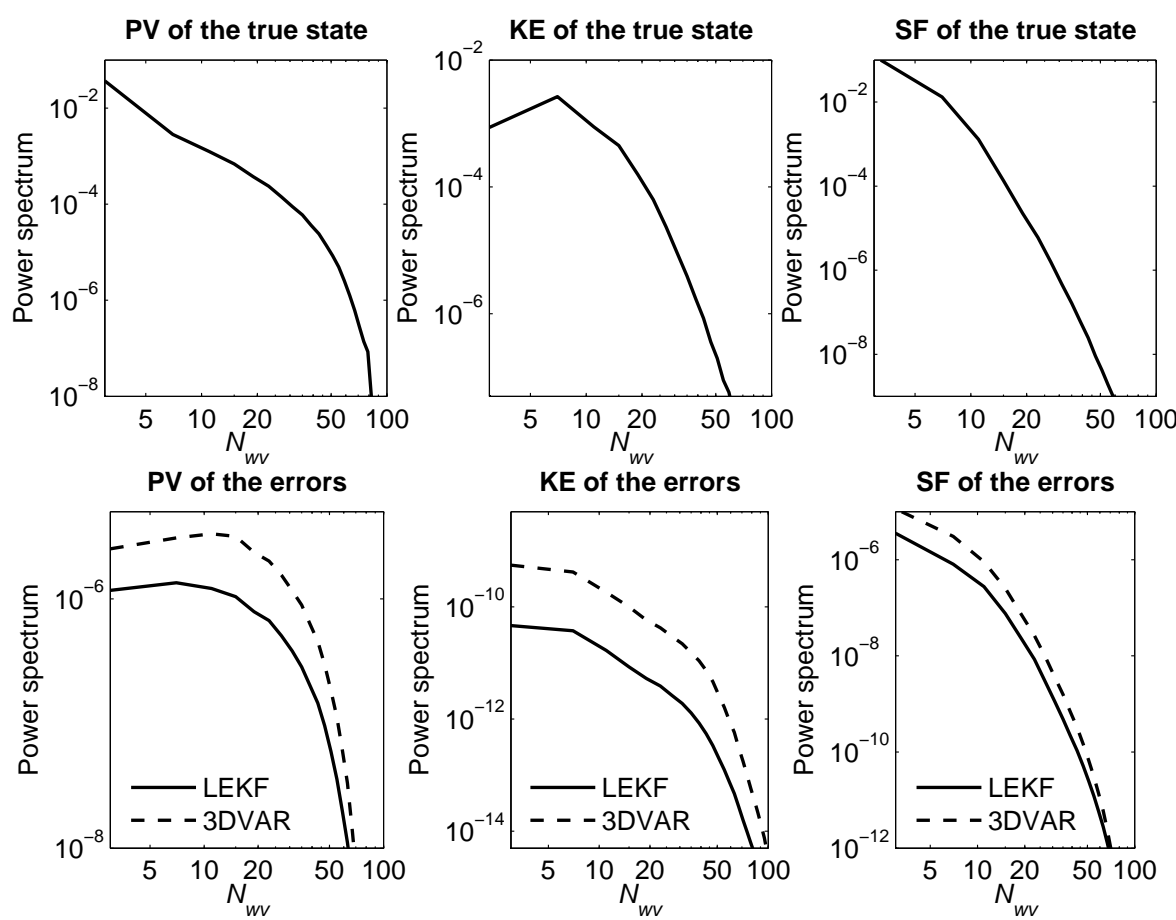

Fig. 6. Power spectrum of the true state (top panels) for potential enstrophy, kinetic energy and streamfunction, respectively. The same quantities for the analysis errors of 3D-Var and LEKF are represented in the bottom panels. Figures are computed using data from step 361 to step 720 (180 days).

amplitude, corresponds to the first part of the simulation, that can be considered a transition phase (see Figs. 1a and b). A large part of the improvement (about $40 \%$ reduction in rms error) is due to the reduction of the error maxima. These results are similar for the space averaged analysis errors in streamfunction at midlevel reported in Fig. 4b. For this variable the improvement is even larger than for potential vorticity: the larger error peaks are significantly reduced, and the relative average improvement in the rms error is about $50 \%$. In Fig. 4c the scatter plot of the forecast errors for the $72 \mathrm{~h}$ forecast is shown for LEKF and 3Dvar. It is important to notice that the improvement gained at the analysis is maintained during the forecast (Fig. 5), further confirming that the errors corrected by the LEKF are those responsible for the large growth of forecast errors. In Fig. 1 it can be seen that the peaks in analysis errors and $48 \mathrm{~h}$ forecast errors occur at the same time. This indicates that fast growing "errors of the day" are present in the initial conditions $48 \mathrm{~h}$ before attaining their large amplitude, and that the LEKF evidently succeeds in correcting these small amplitude, fast growing analysis errors before they have a chance to grow into the forecast.

The top panels of Fig. 6 represent the power spectrum coefficients for potential enstrophy, kinetic energy and streamfunction computed from the true state, plotted against the global wavenumber. Following Morss et al. (2001) the global wavenumbers are computed as
$N_{W V}=\left((2.5 \times k)^{2}+(5.2 \times .5 \times l)^{2}\right)^{\frac{1}{2}}$, where $k$ is the zonal wavenumber and $l$ is the meridional half wavenumber, while the coefficients 2.5 and 5.2 are introduced in order to make the global wavenumber comparable to that of the "real" global atmosphere. The distributions of the power spectrum coefficients of the three quantities are similar. Most of the spectrum amplitude is concentrated at wavenumbers smaller than 20. The corresponding spectrum coefficients for the analysis errors are shown in the bottom panels of Fig. 6 . LEKF has smaller errors than 3D-Var for all wavenumbers and shows a very large improvement for all scales in the kinetic energy field. The improvement for potential vorticity is distributed particularly in mid to small scales while the improvement in the streamfunction appears at the larger scales. The large improvement in the KE field benefits directly from the fact that the assimilation variables are $u, v$, and $T$ (Sect. 3). The 3D-Var background covariance matrix is able to successfully correct large wavelengths, but its performance becomes much worse as the wavenumber increases. This is due to the fact that information on small scales in 3D-Var background error covariance is lost in the statistical average. By contrast, LEKF is able to reduce errors at all wavelengths, so that its error spectrum is similar to the spectrum of the true state. The forecast error is dominated by local structures, and the results suggest that the background error covariance in LEKF represents well these errors. 


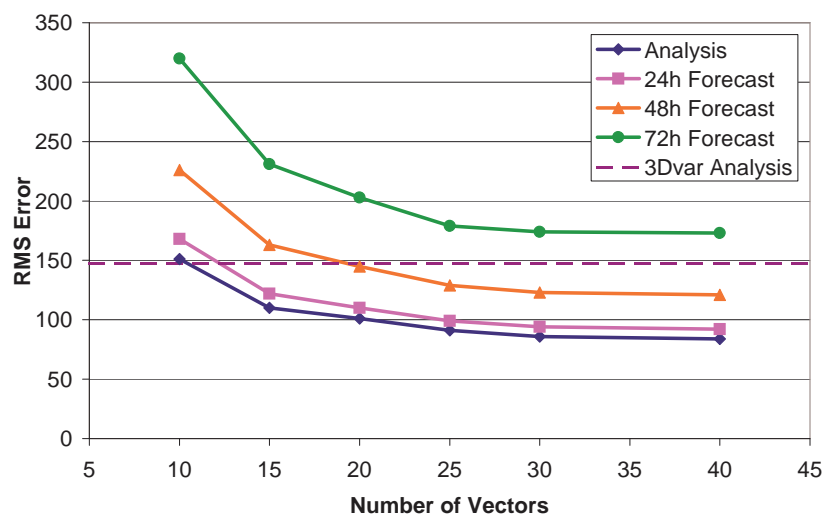

(a)

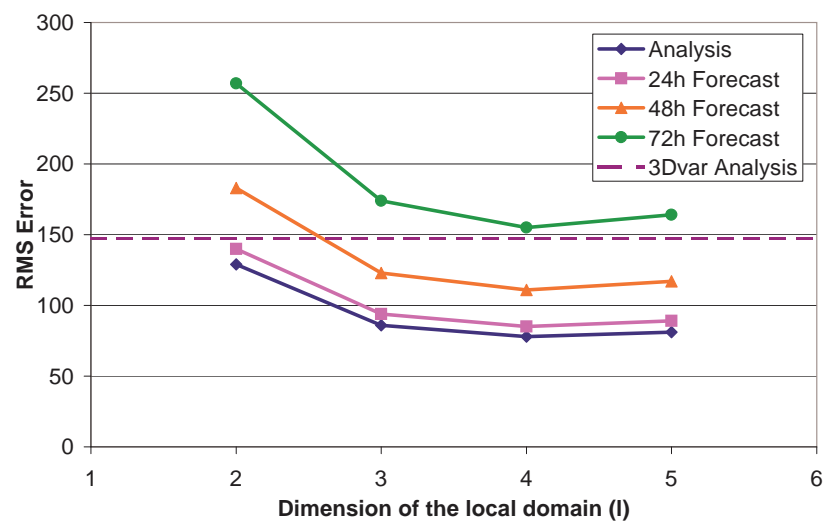

(b)

Fig. 7. (a): Analysis and Forecast Errors averaged in space and time for potential vorticity at midlevel for the LEKF system as the number of vectors used in the ensemble varies. Cases for 10, 15, 20, 25, 30 and 40 vectors are reported. (b): same as (a) but the Forecast Errors are shown as the horizontal dimension of the local domains varies. Cases for $l=2,3,4$, and 5 are reported. In both figures 3DVar Analysis error is shown for reference.

\subsection{Sensitivity of the LEKF to choice of parameters}

As could be expected, LEKF results are sensitively dependent on the number of vectors forming the ensemble. In Fig. 7a the error averaged in space and time for potential vorticity at midlevel is shown for the LEKF system running with $10,15,20,25,30$ and 40 vectors, using a fixed size of the local domain ( $l$ equal to 3 ). The results with $3 \mathrm{D}$-Var are also shown for reference. It can be seen that for this system 10 vectors are not sufficient to improve upon 3D-Var. The performance of the LEKF improves with the number of ensemble members but only up to 30 (chosen as reference in the previous results). The reduction of errors with ensemble size converges and no further significant improvements are observed with 40 members. An ensemble of 30 members has much fewer degrees of freedom than the model, and supports the hypothesis that the local subspace of the most unstable modes is small and that the LEKF could be used for operational purposes with a reasonable computational effort.

Another important parameter in the LEKF is the size of the local volumes ("patches") used in the localization. Figure $7 \mathrm{~b}$ shows the analysis and forecast errors for different patch sizes $(1=2,3,4,5$, corresponding to squares of $5 \times 5$, $7 \times 7,9 \times 9$ and $11 \times 11$ grid points respectively), using an ensemble of 30 members. The analysis errors improve with size up to $1=4(9 \times 9$ grid points $)$, and then they become slightly worse. This can be explained by the fact that when the number of points of each local domain increases, the local dimension of the system also increases, and the number of vectors required to describe well the local instabilities of the system eventually needs to be larger. In other words, the larger the local dimension, the larger the sampling errors in the representation of the background errors with a limited number of ensemble perturbations.
This interpretation is supported by an analysis of the ability of the ensemble of vectors to describe the background error. In Fig. 8 the error variance explained by the ensemble (relative amplitude of the projection of the background error onto the subspace spanned by the ensemble of vectors) is shown. This is done by projecting at each local domain the local vector of the values of the error in potential vorticity onto the subspace spanned by the local vectors representing the $n$ members of the ensemble. The ratio between the amplitude of this projection and the total amplitude of the error is computed, and then averaged over all the local domains and represented in the plots. The process is then repeated at each data assimilation step. Two cases are shown: in Fig. 8a the variation of the projected component is represented using a fixed dimension of the local domain with a varying number of vectors. In Fig. $8 \mathrm{~b}$ the number of vectors is fixed and the size of the local domains is varied. The percentage of the error represented by the ensemble is on average of the order of 90\% and it increases with the number of ensemble members and decreases with the dimension of the local domain.

It is interesting to see how the ensemble's ability to explain the background error depends on the amplitude of the error. In Fig. 9, the relative amplitude of the projection of the background error at a fixed time (the same as in Figs. 2a, 2b, and 3 ) is compared with the total amplitude of the error (contour plot). It clearly shows that the percentage of the vector projected onto the subspace varies substantially in space and that the larger the error, the better its projection on the ensemble. This important property of the LEKF indicates that the projection of the largest errors of the system onto the ensemble is even larger than what is suggested by the average values shown in Fig. 8, and explains the success of the LEKF in reducing the analysis errors even with relatively few ensemble members (in agreement with Szunyogh et al., 2005) . 


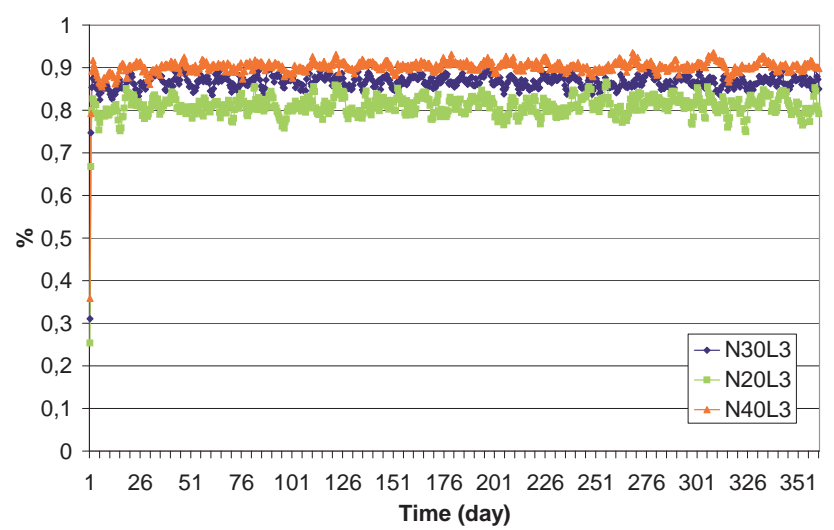

(a)

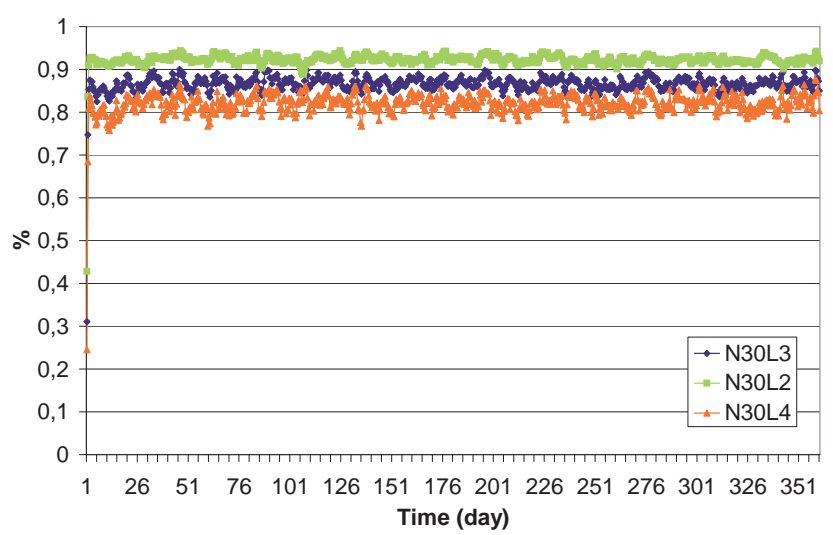

(b)

Fig. 8. Time evolution of projection of the background error in potential vorticity on the subspace spanned by the ensemble of local vectors. (a): The dimension of the local domain is constant $(l=3)$ and the number of vectors varies $(N=20,30,40)$. (b): The number of vectors is constant $(N=30)$ and the dimension of the local domain varies $(l=2,3,4)$.

The sequential methods for assimilation of observations (Houtekamer and Mitchell, 1998; Tippett et al., 2002), use a "localization" of the error covariance obtained by multiplying the correlation between grid points and observation points forecast errors by a Gaussian function of the distance between these points. This has the effect of reducing the covariance sampling errors at long distances. By contrast, the LEKF uses a step function (1 within the local domain and zero outside). Miyoshi (2005) found that this made the LEKF slightly worse than the Ensemble Square Root Filter (EnSRF) of Whitaker and Hamill for the SPEEDY global model (Molteni, 2003). He followed a suggestion of Hunt (2005) to apply instead the inverse of the Gaussian localization operator, to the $\mathbf{R}$ matrix, in such a way that:

$\tilde{\mathbf{R}}=e^{\frac{\left(\boldsymbol{r}-\boldsymbol{r}_{o}\right)^{2}}{2 \sigma^{2}}} \circ \mathbf{R}$,

where $\sigma$ is of the order of $l, \boldsymbol{r}-\boldsymbol{r}_{o}$ is the distance from the center of the domain and the symbol $\circ$ indicates a Shur (or Hadamard) product. The effect of this is to increase the effective observation error for observations far away from the central analysis point, achieving the same result as with the standard localization. We tested this approach and found a very small improvement (about $2 \%$ ) when using the "multicolumn" approach.

Finally we discuss the vertical localization of the domain. In all the experiments performed in the framework of this work the local domain has been computed including all the vertical levels of the model without vertical decomposition of the domain. Nevertheless, it is interesting to explore whether further improvements could be expected from vertical decomposition of the local domain. For this decomposition to be useful, it is necessary to prove that there is not a high correlation between the background errors at different levels. In Table 2 the average vertical correlation between the background error at each level of the local domain is shown. The correlation is computed considering each local domain and then averaged in space and time. The values presented in Table 2 do not depend significantly on the detailed implementation of the system. Results clearly show a significant correlation between each level and its closest neighbors, while the correlation with the second neighbor and levels further away is much smaller. This is a clear indication that for an optimal configuration of this system vertical localization should be introduced with local domains based on 3 vertical levels.

\section{Summary and conclusions}

In this work we have implemented the Local Ensemble Kalman Filter (LEKF) described in Ott et al. (2004) for a quasi-geostrophic model widely used in the literature. Results obtained with the LEKF have been compared to those obtained by means of an optimized version of a 3D-Var, showing a marked improvement both in the analyses and the forecasts. As pointed out in the introduction, the optimization of the 3D-Var did not include an attempt to generate a flow dependent background error covariance matrix $\mathbf{B}$, which could have reduced the improvement of the LEKF. A more general comparison between different data assimilation systems, including 4D-Var and hybrid systems, is presented in Yang et al. (2007, see footnote 1 at page 95). In order to adapt to the local framework of LEKF in the presence of model global spectral transforms, we proposed an alternative split of the observation operator that can be easily implemented in operational setups.

Different configurations of the LEKF system have been considered and compared to the 3D-Var. With an ensemble of 30 vectors, best results have been obtained using a horizontal 


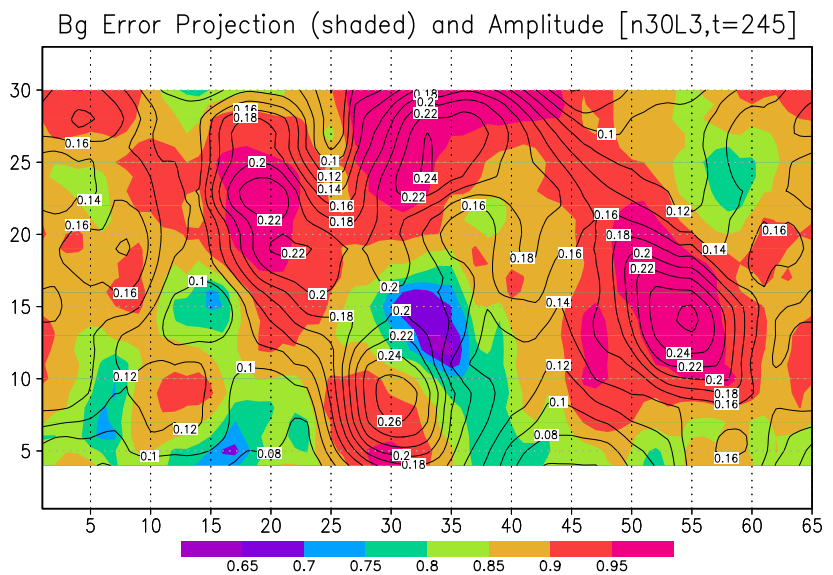

Fig. 9. Example showing the relationship between the percentage projection of the background error onto the subspace spanned by the local vectors (colors) and the background error (contours). The time chosen is the same of Figs. $2 \mathrm{a}$ and $\mathrm{b}$ and 3 ; the number of vectors used is $n=30$ and the dimension of the local domain is of $7 \times 7 \times 5$ grid points $(l=3)$.

dimension of the local domains of $9 \times 9$ grid points, which allows for the inclusion of a sufficient number of observations, leaving the dimension of the local space small enough to be described by a small set of vectors. The fact that, using a relatively small ensemble, an optimal value of the horizontal dimension of the local domain does exist is an indication of the need to find a compromise between including the maximum observational information, and limiting the dimension of the local vectors in order to let the ensemble member provide an optimal description of the instabilities of the system. By contrast, using a fixed local domain $(l=3)$, results show a monotonic improvement as the number of ensemble members increases, with saturation at about 30 members indicating that a relatively small ensemble is sufficient to provide a good description of the instabilities and therefore of the errors.

In order to get stable results, it is necessary to prevent the forecast perturbations from collapsing with time into a subspace which is too small. For the quasi-geostrophic model, the use of additive random perturbations after each data assimilation step gives much better results than multiplicative inflation, which in fact fails to prevent filter divergence.

For this system the introduction of the localization of the observational error covariance matrix $\mathbf{R}$ tested by Miyoshi (2005), based on a suggestion of Hunt (2005), does not result in a significant improvement. This result may not be general and may be associated with the fact that we obtained best simulations by means of the "multi column" method, so that the contribution of the localization is less important than in the case of the "central column update" method.

It is important that the entire relative improvement obtained for the analysis is maintained throughout the 3-day
Table 2. Time and space average of vertical correlation of the background errors in potential vorticity at different levels of the local domain. Level 1 represents the bottom level, level 5 the top level.

\begin{tabular}{rrrrrr}
\hline Level & 1 & 2 & 3 & 4 & 5 \\
\hline 1 & 1.00 & 0.46 & 0.17 & 0.10 & 0.10 \\
2 & 0.46 & 1.00 & 0.35 & 0.17 & 0.14 \\
3 & 0.17 & 0.35 & 1.00 & 0.47 & 0.33 \\
4 & 0.10 & 0.17 & 0.47 & 1.00 & 0.63 \\
5 & 0.10 & 0.14 & 0.33 & 0.63 & 1.00 \\
\hline
\end{tabular}

forecasts, indicating that the system is indeed correcting the analysis errors laying in the directions of the fast growing modes.

The vertical correlation of background errors (Table 2) shows that the correlation quickly drops beyond the closest level. This suggests that the LEKF system used in this work could be improved by introducing a vertical localization of the domain, in particular considering local domains based on 3 vertical levels.

Our results are very encouraging, indicating that LEKF is much more accurate than 3D-Var even with a rather small ensemble size. Some areas that still require further study are:

1. the use of a flow-dependent amplitude of the additive random perturbations;

2. the performance of the system during the initial spinup, especially if the starting point of the simulation is far from the true state of the flow;

3. the relationship between additive or multiplicative inflation and the observational density, in particular when the observational network is not homogeneous.

Acknowledgements. We are grateful to the University of Maryland Chaos/Weather group, and in particular to E. Ott for suggesting the application of the LEKF to the QG model. Rebecca Morss kindly made available the model and 3D-Var system. This research was partially supported by the European Project WERMED, Interreg IIIb.

Edited by: Z. Toth

Reviewed by: two referees

\section{References}

Anderson, J. S.: An ensemble adjustment filter for data assimilation, Mon. Wea. Rev., 129, 2884-2903, 2001.

Annan, J. D.: On the orthogonality of Bred Vectors, Mon. Wea. Rev., 132, 843-849, 2004.

Bennett, A. F., Chua, B. S., and Leslie, L. M.: Generalized inversion of a global NWP model., Meteorol. Atmos. Phys., 60, 165-178, 1996. 
Corazza, M., Kalnay, E., Patil, D. J., Ott, E., Yorke, J., Szunyogh, I., and Cai, M.: Use of the breeding technique in the estimation of the background error covariance matrix for a quasigeostrophic model, in: AMS Symposium on Observations, Data Assimilation and Probabilistic Prediction, pp. 154-157, Orlando, Florida, 2002.

Corazza, M., Kalnay, E., Patil, D. J., Yang, S.-C., Morss, R., Cai, M., Szunyogh, I., Hunt, B. R., and Yorke, J. A.: Use of the breeding technique to estimate the structure of the analysis "errors of the day", Nonlin. Processes Geophys., 10, 233-243, 2003, http://www.nonlin-processes-geophys.net/10/233/2003/.

Courtier, P., Thèpaut, J.-N., and Hollingsworth, A.: A strategy for operational implementation of 4D-Var, using an incrementeal approach, Quart. J. Roy. Meteorol. Soc., 120, 1367-1387, 1994.

Daley, R.: Atmospheric data analysis, Cambridge University Press, New York, 1993.

De Pondeca, M. S. F. V., Purser, R. J., Parrish, D. F., and Derber, J. C.: Comparison of strategies for the specification of anisotropies in the covariances of a three-dimensional atmospheric data assimilation system, NOAA/NCEP Office Note 452, 13 pp, 2006.

Etherton, B. J. and Bishop, C. H.: Resilence of Hybrid Ensemble/3DVAR Analysis Schemes to Model Error and Ensemble Covariance Error, Mon. Wea. Rev., 132, 1065-1080, 2004.

Evensen, G.: Sequential data assimilation with a nonlinear quasigeostrophic model using Monte Carlo methods to forecast error statistics, J. Geophys. Res., 99, 10 143-10 162, 1994.

Hamill, T. M. and Snyder, C.: A Hybrid Ensemble Kalman Filter3D Variational Analysis Scheme, Mon. Wea. Rev., 128, 29052919, 2000.

Houtekamer, P. L. and Mitchell, H. L.: Data assimilation usin an ensemble Kalman filter technique, Mon. Wea. Rev., 126, 796$811,1998$.

Houtekamer, P. L., Mitchell, H. L., Pellerin, G., Buehner, M., Charron, M., Spacek, L., and Hansen, B.: Atmospheric Data Assimilation with an Ensemble Kalman Filter: Results with Real Observations, Mon. Wea. Rev., 133, 604-620, 2005.

Hunt, B. R.: Efficient Data Assimilation for Spatiotemporal Chaos: a Local Ensemble Transform Kalman Filter, http://www.citebase. org/cgi-bin/citations?id=oai:arXiv.org:physics/0511236, 2005.

Kalnay, E.: Atmospheric Modeling, Data Assimilation and Predictability, Cambridge University Press, 340pp., 2003.

Kalnay, E. and Toth, Z.: Removing growing errors in the analysis cycle, in: Tenth Conference on Numerical Weather Prediction, pp. 212-215, Amer. Meteorol. Soc., 1994.

Klinker, E., Rabier, F., Kelly, G., and Mahfouf, J.-F.: The ECMWF operational implementation of four-dimensional variational assimilation. III: Experimental results and diagnostics with operational configuration, Quart. J. Roy. Meteorol. Soc., 126, 11911216, 2000.

Lorenc, A. C.: Analysis methods for numerical weather prediction, Quart. J. Roy. Meteorol. Soc., 112, 1177-1194, 1986.

Miyoshi, T.: Ensemble Kalman Filter Experiments with a primitive equation global model, Ph.D. thesis, University of Maryland, College Park, MD, 2005.
Molteni, F.: Atmospheric simulations using a GCM with simplified physical parametrizations. I: model climatology and variability in multi-decadal experiments., Clim. Dyn., 20, 175-191, 2003.

Morss, R. E.: Adaptive observations: Idealized sampling strategies for improving numerical weather prediction, Ph.D. thesis, Massachusetts Institute of Technology, 225 pp., 1998.

Morss, R. E., Emanuel, K. A., and Snyder, C.: Idealized adaptive observation strategies for improving numerical weather prediction, J. Atmos. Sci., 58, 210-234, 2001.

Oczkowski, M., Szunyogh, I., and Patil, D. J.: Mechanisms for the Development of Locally Low-Dimensional Atmospheric Dynamics, J. Atmos. Sci., 62, 1135-1156, 2005.

Ott, E., Hunt, B. R., Szunyogh, I., Corazza, M., Kalnay, E., Patil, D. J., and Yorke, J. E.: Exploiting low-dimensionality of the atmospheric dynamics for efficient ensemble Kalman Filtering, http://arXiv:physics/0203058, 2002.

Ott, E., Hunt, B. R., Szunyogh, I., Zimin, A. V., Kostelich, E. J., Corazza, M., Kalnay, E., Patil, D. J., and Yorke, J.: A local ensemble Kalman filter for atmospheric data assimilation, Tellus, 56A, 415-428, 2004.

Parrish, D. F. and Derber, J. C.: The National Meteorological Center's Spectral Statistical-Interpolation Analysis System, Mon. Wea. Rev., 120, 1747-1763, 1992.

Patil, D. J. S., Hunt, B. R., Kalnay, E., Yorke, J. A., and Ott, E.: Local Low Dimensionality of Atmospheric Dynamics, Phys. Rev. Lett., 86, 5878-5881, 2001.

Purser, R. J.: A geometrical approach to the synthesis of smooth anisotropic covariance operators for data assimilation, NOAA/NCEP Office Note 447, 60 pp, 2005.

Rabier, F., Jarvinen, H., Klinker, E., Mahfouf, J.-F., and Simmons, A.: The ECMWF operational implementation of fourdimensional variational physics, Quart. J. Roy. Meteorol. Soc., 126, 1143-1170, 2000.

Rotunno, R. and Bao, J. W.: A case study of cyclogenesis using a model hierarchy, Mon. Wea. Rev., 124, 1051-1066, 1996.

Szunyogh, I., Kostelich, E. J., Gyarmati, G., Hunt, B. R., Ott, E., Zimin, A. V., Kalnay, E., Patil, D. J., and Yorke, J. A.: A local ensemble kalman filter for the NCEP GFS model, no. 9.4 $\mathrm{J}$ in Proceedings of the AMS meeting, Seattle, Washington, 2004.

Szunyogh, I., Kostelich, E. J., Gyarmati, G., Patil, D. J., Hunt, B. R., Kalnay, E., Ott, E., and Yorke, J. A.: Assessing a local ensemble Kalman filter: Perfect model experiments with the National Centers for Environmental Prediction global model, Tellus, 57A, 528-545, 2005.

Tippett, M. K., Anderson, J. L., Bishop, C. H., Hamill, T. M., and Whitaker, J. S.: Ensemble square-root filters, Mon. Wea. Rev., 131, 1485-1490, 2002.

Toth, Z. and Kalnay, E.: Ensemble forecasting at NMC: the generation of perturbations, Bull. Amer. Meteorol. Soc., 74, 23172330, 1993.

Toth, Z. and Kalnay, E.: Ensemble forecasting at NCEP: the breeding method, Mon. Wea. Rev., 125, 3297-3318, 1997.

Wang, X. and Bishop, C. H.: A comparison of Breeding and Ensemble Transform Kalman Filter Ensemble Forecast Schemes, J. Atmos. Sci., 60, 1140-1158, 2003. 